Rapid Reviews COVID-19

\title{
Review 1: "Qualitatively \\ distinct modes of Sputnik \\ $V$ vaccine-neutralization \\ escape by SARS-CoV-2 \\ Spike variants"
}

\author{
Alexander Mentzer ${ }^{1}$ \\ ${ }^{1}$ University of Oxford Hooke Library: University of Oxford, Wellcome Trust Centre for human \\ genetics
}

Published on: May 29, 2021

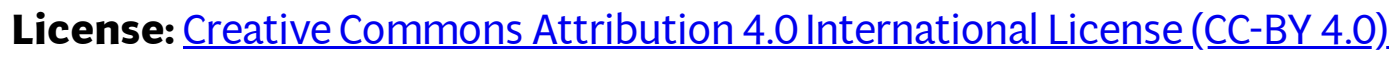




\section{$\underline{\text { RR:C19 Evidence Scale rating by reviewer: }}$}

- Potentially informative. The main claims made are not strongly justified by the methods and data, but may yield some insight. The results and conclusions of the study may resemble those from the hypothetical ideal study, but there is substantial room for doubt. Decision-makers should consider this evidence only with a thorough understanding of its weaknesses, alongside other evidence and theory. Decisionmakers should not consider this actionable, unless the weaknesses are clearly understood and there is other theory and evidence to further support it.

*****************************************

\section{Review:}

Summary of the study:

There is a desperate need to understand the likelihood that novel variants of the SARSCoV-2 virus will escape vaccine-induced immunity derived from Victoria/Wuhan strain Spike protein. Recent literature highlights the importance of specific variants including E484K that may inflict such escape, especially if the vaccine construct lacks the prefusion conformation K986P and V987P substitutions. There has been a substantial amount of recent literature presenting the likelihood that variants harboring E484K, such as the B.1.351 variant, will escape immunity induced by the Pfizer/BioNTech, AZ/Oxford, and Moderna vaccines. Despite substantial insights afforded by this literature, there has been substantially less work on other vaccines including the Sputnik V vaccine (Gam-COVID-Vac).

In this preprint, the authors aimed to investigate the likelihood that E484K variants escape Gameyla Sputnik V vaccine-induced immunity. The authors utilize the increasingly deployed rcVSV-CoV2-S system (replication-competent VSV expressing SARS-CoV-2 spike in place of VSV-G) to express E484K Spike variants. They have, however, modified the system to avoid extensive end-stage passaging that may incur novel, unwanted, and unanticipated variants into Spike. They then measured IC50 against multiple standard Spike variants using samples from 12 Sputnik V vaccine recipients recruited in Argentina. Using a variety of in vitro model analyses the authors claim that vaccine escape will be more likely with B.1.351 Spike variant over and above WT or B.1.1.7 variants.

Specific Comments: 
This work represents a helpful addition to the literature that builds on our knowledge of potential variant-vaccine escape focusing on other vaccines that have largely been neglected including specifically the Gameyla Sputnik V that is being deployed in a significant number of countries. Although the approach is not novel and the findings are not unexpected, the focus on this other vaccine and the use of samples from other countries is important to present.

The study does have some limitations that should be addressed before it conclusions can be more reliably interpreted. Firstly, although the author's novel approach to the rcVSV-CoV2-S system is theoretically respectable and the results look plausible, the significant adjustment to the protocol does warrant further validation. It would be helpful to have a comparison of results against standard SARS-CoV-2 neutralization at least against a single variant of the virus, perhaps Victoria or B.1.1.7 which are now widely available. Secondly, given that the focus of the paper is on countries that are deploying the Gameyla Sputnik V vaccine (e.g. Argentina) it would be desirable and most elegant to include variants that are specific to those countries, especially if their system is so versatile. Thirdly, the information on their included participants is limited. Much of their interpretation of patterns of neutralization assumes that individuals have not been exposed to any naturally circulating viral variants but the authors do not state whether the participants have any history of prior exposure to SARS-CoV-2 or COVID disease.

Finally, even with these adjustments, there will still be limitations to the informativeness of the data. Ultimately, determining the likelihood of escape requires clinical follow-up of patients receiving the vaccine rather than in vitro neutralization assays. Furthermore, the nature of the pseudovirus neutralization assay using different viral constructs will always come with questions of representativeness of in vivo neutralization.

\section{Conclusions:}

This study provides some preliminary evidence that SARS-CoV-2 variants, and B.1.351 in particular, may escape Gameyla Sputnik V vaccination-induced immunity but there are significant limitations that require addressing and consideration before reliably interpreting the results presented. 